\title{
Implementation of Hybrid Algorithm for Mobility and Routing Issues in MANET
}

\author{
Vinita Ratnaparkhi \\ CSE Department Lakshmi \\ Narain College of Technology \\ Indore, India
}

\author{
Pawan Patidar \\ Asst. Prof. in CSE Department \\ Lakshmi Narain College of \\ Technology Indore, India
}

\author{
M.K. Rawat, Ph.D \\ Prof. in CSE Department \\ Lakshmi Narain College of \\ Technology Indore, India
}

\begin{abstract}
In this paper, the problem of dynamic multicast routing in mobile ad hoc networks is investigated. Dynamic multicast routing has become a major issue in Manet. Mobile ad hoc networks (MANETs) are collection of wireless mobile nodes, thus connectivity is an issue in this network. Moreover it, the network topology is defined by its routing strategy. In MANET, the network topology keeps changing due to its inherent characteristics such as node mobility. Mobility is the main factor affecting topology changes and route formation. Routing algorithms are responsible for discovering routes between source and destination, in independent mobility environment. Furthermore, this kind of network organization performance is an essential issue.

In the previous algorithm [1] the multi-casting tree is improved by using genetic algorithm based high-low mutation genetic algorithm. The proposed algorithm (hybrid algorithm), extension of the previously available algorithm (High low hyper mutation genetic algorithm) is implemented using the network simulator 3 environment. Hybrid algorithm is a mixture of genetic algorithm and K-nearest neighbor algorithm. Then hybrid algorithm compares with traditional algorithm using various performance parameters like throughput, packet delivery ratio, packet drop ratio, end to end delay.
\end{abstract}

\section{General Terms}

Mobility, dynamic multicast routing problem, genetic and kNearest Neighbour algorithm, hyper mutation based genetic algorithm.

\section{Keywords}

MANET, Routing, Genetic algorithm, k-Nearest Neighbour, high low hyper mutation genetic algorithm.

\section{INTRODUCTION}

Mobile ad hoc network are collection of wireless mobile nodes that are communicating in infrastructure less network. These nodes are connected with the wireless links, in independent mobility environment. So, the network nodes are uses the cooperative communication. The proposed work in this network is based on the concept of routing issues. This proposed routing algorithm (hybrid algorithm) is enhancement of the traditional routing genetic algorithm (high low hyper mutation genetic algorithm).

MANET (mobile ad hoc networks) is a new generation technology. In this type of network not any infrastructure is available consequently network nodes are not connected through a wired link. And nodes are able to move any direction independently.
So mobility is a primary characteristic of this type of network Mobility is the main factor affecting topology of the network and route formation. The mobility of the nodes affects the number of average connected paths, which in turn affect the performance of the routing algorithm. Therefore required to find a new strategy by which the mobility of network is handled efficiently. Due to this property MANET involves a wide verity of the applications such as the military applications, during natural disasters and others. But technically handling and managing the mobility is a complex issue in MANET.

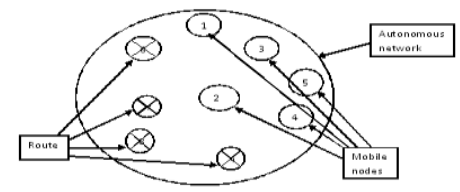

Figure 1: Manet Diagram

As given in Figure 1 Autonomous network using wireless mobile nodes and routes. The proposed work is intended to handle the mobility and providing the efficient way of dealing with their performance.

In addition of that here the dynamic multicast routing problem is investigated and a new solution is suggested. Multicast is the sending of a packet from one sender to multiple receivers with a single send operation. The multicast routing problem involves additional combinatorial optimization problem. In mobile ad hoc networks, the topology of the network frequently changes due to its inherent characteristics such as mobility. Hence, the dynamic multicast routing problem in MANETs turns out to be a dynamic optimization problem (DOP). An effective multicast routing algorithm keeps track the topological changes and adapt the best multicast tree to the topological changes accordingly. Therefore in this proposed work solve the problem of dynamic multicast routing problem and mobility issues are discovered and performance of the network increases using various quality of service parameter like throughput, packet delivery ratio, packet drop ratio, end to end delay. At the predefined high level and for the second half, the mutation rate is set at the pre-defined low level.

\section{BACKGROUND}

\section{1 High low mutation process}

Mutation is an operator used in genetic algorithm for managing genetic diversity from current generation to the next generation. It is similar to biological mutation that alters one or more genes in a chromosome from its initial state. During the mutation the existing solution may change from the previously defined 
solutions. Therefore GA can able to generate better solution using mutation.

DOPs (dynamic optimization problem), commonly becomes a big problem for Gas. Because Gas (genetic algorithm) can quickly adopt environmental changes and maintain population diversity and their adaptability. Hypermutation based Gas is also a basic technique that enables to cope with the dynamically changing environments. In traditional hyper mutation genetic algorithm, the mutation rate is fixed when the entire evolutionary process. Though, the hyper-mutation is to adjust the mutation rate adaptively. When environmental changes arise, then for maintain the population diversity mutation rate should be increased to a high level. When the environment is in a steady state, then the mutation rate should be decreased to a low level. Subsequently mutation can help to generate new solutions.

Following two different types of hyper-mutation schemes:

1) high low hypermutation.

2) gradual hyper-mutation.

In the high low hyper-mutation, change interval is defined. Number of generations between two consecutive changes is called change interval. For the first half of the change interval, the mutation rate is set.

In the gradual hyper-mutation, the mutation rate is increased to the pre-defined high level when there is a change to the environment. Then at each generation the mutation rate is gradually decreased till it reaches the predefined low level or another change arrives.

\subsection{Genetic Algorithm}

Genetic algorithm is one of the popular approaches for making search for large amount of data. The bio informatics knowledge is used to find the fittest answers in number of repetitive or iterative calculations. So first we discuss the primary functioning of the genetic algorithm. The genetic algorithms use the three main principles of the natural evolution: reproduction, natural selection and diversity of the species.

Genetic Algorithms works with a set of individuals and representing possible solutions of the tasks. The selection principle is applied by using a criterion, giving selective evaluation for the individual with respect to the desired solution and the best-suited individuals create the next generation.

Generate initial population -the algorithms in first generation randomly generated by selecting the genes of the chromosomes among the allowed alphabet for the gene because of the conventional computational procedure it is accepted that, all populations must have the same number $(\mathrm{N})$ of individuals, in these values of the function that we want to minimize of maximize.

Check for termination of the algorithm - as in the most optimization algorithms, it is possible to stop the genetic optimization by: Value of function, highest number of iterations and Stall generation

Selection - between all individuals in the current population are chose those, who will continue and by means of crossover and mutation will produce offspring population. At this stage elitism could be used - the best $\mathrm{n}$ individuals are directly transferred to the next generation.
Crossover - basically crossover is the process of the individuals chosen by selection process recombine with each other and new individuals will be created. The goal is to get child offspring individuals, which is inheriting the best possible combination of the characteristics (genes) of their parents.

Mutation - mutation is a process of random change of some of the genes of individuals. It is guaranteed that even if none of the gene contain the required solution of gene value for the extreme, it is still possible to reach the solution of gene.

New generation - the elite individuals chosen from the selection are combined with those who passed the crossover and mutation, and form the next generation.

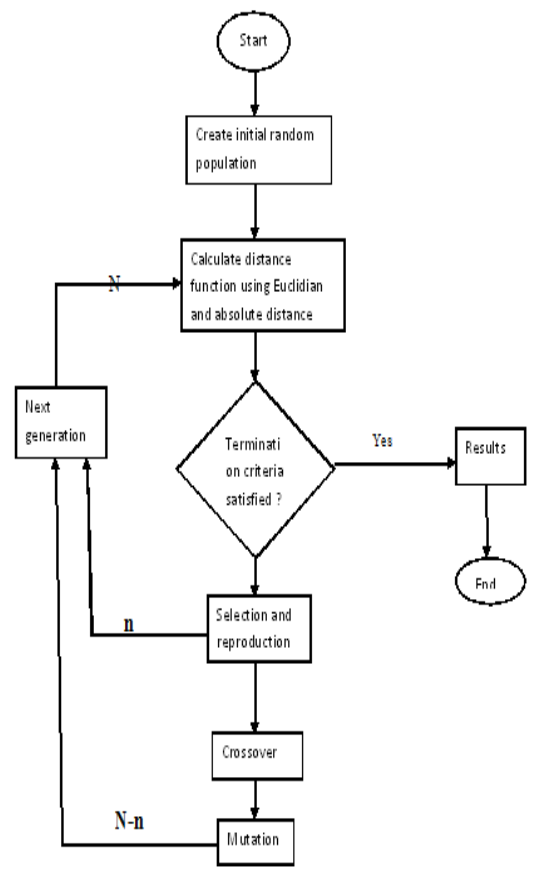

Figure 2: Basic Flow diagram of proposed hybrid genetic algorithm

\section{3 k-Nearest-Neighbour (k-NN) algorithm}

The K-nearest-neighbour algorithm measures the distance between a query scenarios in the data base. Calculate the distance between these two scenarios using a distance function $\mathrm{d}(\mathrm{x}, \mathrm{y})$, where $\mathrm{x}, \mathrm{y}$ are scenarios.

In which calculate two types of distance function such as:

- absolute distance

- Euclidean distance measuring

The overall KNN algorithm is running in the following steps:

1. Store the output values of the $M$ nearest neighbors to query scenario $Q$ in vector $r=\left\{r_{1}, \ldots \ldots, r_{m}\right\}$ by repeating the following loop $\mathrm{M}$ times:

a. Go to the next scenario $S_{i}$ in the data set, where $I$ is the current iteration within the domain $\{1 \ldots \ldots . \mathrm{P}\}$

b. If $\mathrm{Q}$ is not set or $\mathrm{q}<\mathrm{d}\left(\mathrm{q}, \mathrm{S}_{\mathrm{i}}\right)$ : $\mathrm{q} d\left(\mathrm{q}, \mathrm{S}_{\mathrm{i}}\right), \mathrm{t}_{\mathrm{i}}$

c. Loop until we reach the end of the data set. 
d. Store $\mathrm{q}$ into vector $\mathrm{c}$ and $\mathrm{t}$ into vector $\mathrm{r}$.

2. Calculate the arithmetic mean output across $r$ as follows:

- Return $r$ as the output value for the query scenario $q$.

\section{WORK DONE}

\subsection{High low hyper mutation genetic algorithm}

According to the found description in [16] the classical genetic algorithm can described using the below given genetic pseudo code.

Input:

instance $\Pi$,

size $\alpha$ of population,

rate $\beta$ of elitism,

rate $\gamma$ of mutation,

number $\delta$ of iterations

Output: solution $X$

// initialization

1. generate $\alpha$ feasible solutions randomly;

2. save them in the population $P \mathrm{OP}$;

//Loop until the terminal condition

3. for $i=1$ to $\delta$ do

//Elitism based selection

4. number of elitism $n \mathrm{e}=\alpha \cdot \beta$;

5. select the best $n$ e solutions in $P_{\mathrm{o}} P$ and save them in $P_{\mathrm{o}} P_{1}$;

//Crossover

6. number of crossover $n \mathrm{c}=(\alpha-n \mathrm{e}) / 2$;

7. for $j=1$ to $n \mathrm{c}$ do

a. randomly select two solutions $X_{A}$ and $X_{B}$ from $P \mathrm{o} P$;

b. generate $X_{C}$ and $X_{D}$ by one-point crossover to $X_{A}$ and $X_{B}$;

c. $\quad$ save $X_{C}$ and $X_{D}$ to $P_{\mathrm{o}} P_{2}$

8. endfor

//Mutation

9. for $j=1$ to $n \mathrm{c}$ do a. $\quad$ select a solution $X_{j}$ from $P_{\mathrm{o}} P_{2}$;

b. mutate each bit of $X_{j}$ under the rate $\gamma$ and generate a new solution $X_{j}{ }^{\prime}$;

c. if $X_{j}^{\prime}$ is unfeasible

i. update $X_{j}^{\prime}$ with a feasible solution by repairing $X_{j}^{\prime}$;

d. end if

e. update $X_{j}$ with $X_{j}^{\prime}$ in $P_{o} P_{2}$;

10. endfor

//Updating

11. update $P \mathrm{o} P=P_{\mathrm{o}} P_{1}+P_{\mathrm{o}} P_{2}$;

12. end for

13. Returning the best solution

14. return the best solution $X$ in $P_{\mathrm{o}} P$;

\subsection{Proposed algorithm}

Genetic algorithm is one of the popular approaches for making search for large amount of data. The bio informatics knowledge is used to find the fittest answers in number of repetitive or iterative calculations. This algorithm is guarantees to produce an optimum solution for the specific problem space. In this search process the initial population is randomly generated. If less population sequences are less than the execution of hybrid algorithm is becomes faster than traditional processing of the genetic algorithm (high low hyper mutation genetic algorithm). Therefore in order to improve the performance of hybrid genetic algorithm required to apply changes in two basic steps:

1. first involves in population evaluation using the below given distance function:

$$
D\left(x_{v} y\right)=\sum_{k=0}^{n}\left|x_{k}-y_{k}\right|
$$

This function returns the most similar values in the generated population; the similar values can be reduced using this function evaluation.

2. In the next step termination condition of algorithm which create by reducing the number of generations. As we know that for finding the next generation populations, initially system generates and evaluates all the possible node combination. But some nodes are not possible in practical network scenarios. So, the unutilized or impossible sequences of nodes are reduced using the distance function. The proposed system can be summarized using the below given steps: 
Input: number of nodes

Outcome: routes

Process:

- Generate initial population

- Calculate distance function for each generated population using

- Remove population having distance less than .5

- Check for termination condition

- $\quad$ Perform selection, cross over, and mutation

- Get new generation

- If new generated sequence having improved fitness value then

- $\quad$ Go to step 3

- $\quad$ Else

- Return the generated sequence

The proposed algorithm is a hybrid algorithm that is designed using genetic algorithm and K-NN algorithm. Where the processing steps are inherited from genetic algorithm and the distance measurement and node elimination process is derived using K-NN algorithm.

\section{EXPERIMENTAL ENVIRONMENT}

\subsection{Hardware and Software Requirement}

The implementation required for this software and hardware on the development side system.

\section{Recommended}

- $\quad$ 2.0 GHz Processor required (Pentium IV and above)

- $\quad$ Minimum 2 GB RAM

- 25 GB hard disk space

\section{Software}

- Operating System (Linux)

- NS3

- $\quad$ GNU Plot

- NETANIMATOR

\subsection{Network simulator 3}

NS-3 is a discrete-event network simulator, which is used for research, educational and development of project. Ns-3 is freely available and distributable under the GNU GPLv2 license to use. The main aim of the NS-3 is to develop a project in open source simulation environment for networking research and development. It is designed for modern networking research with the simulation needs. The NS-3 project is devoted to building simulation environment with well document support. Ns-3 integrates the features as easy to use, debug and wellorganized analysis of the whole simulation workflow. Using the simulation configuration the developers get trace collection.

In addition of that, the NS-3 software supports the simulation prototypes development that is accurate to allow NS-3 to use as a real time network emulator .Using this property NS3 permits to implementation of many existing real-world protocol and that is reused within NS-3 simulation models.

Both IP and non-IP based networks are easily constructed and supportable Using the NS-3 simulation environment. Different types of static or dynamic routing protocols like OLSR and AODV for IP-based applications are also simulated using this simulation environment.

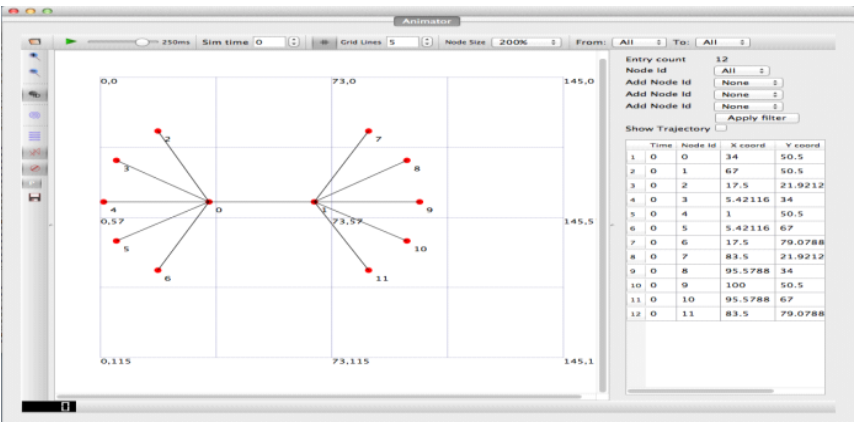

Figure 3: NetAnim

\subsection{Simulation Setup}

Simulation required additional script writing for performing the experiments using python or using $\mathrm{C}++$ scripts. User has freedom to select their-own language for scripting. Once network script is compiled and executed. Than some additional files are generated to get the network information for performance evaluation and animation of network and created scenario. NETANIM can be used for visualization and GNUPLOT is a good utility for use performance graph and results.

To simulate our proposed work we first setup network environment. Then we simulate and compare our proposed techniques in the four scenarios.

Table 1 simulation setup

\begin{tabular}{|l|l|}
\hline Parameters & Description \\
\hline Number of node & $10,20,30,40,50$ \\
\hline Mobility model & RandomWalk2dMobilityModel \\
\hline Simulation time & $50 \mathrm{sec}$ \\
\hline Simulation size & $1000 \times 1000$ \\
\hline
\end{tabular}




\begin{tabular}{|l|l|}
\hline Routing protocol & High-low and Genetic \\
\hline Loss Model & Range propagation \\
\hline Node speed & $20 \mathrm{~m} / \mathrm{s}$. \\
\hline Data Rate & $500 \mathrm{~kb} / \mathrm{s}$ \\
\hline
\end{tabular}

Network simulation having two most important parts first parameters selection and configuring the network simulation environment and second the simulation of network scenarios for performing the experiments and results evaluation.

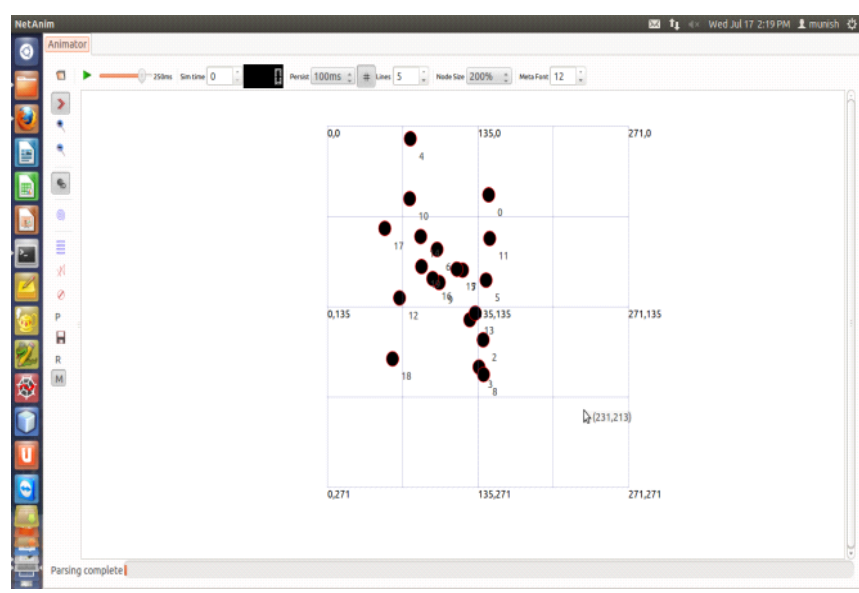

Figure 4: initial network animations

Here two essential scenarios are represented :

- Implementation of MANET using high low mutation protocol: in this network scenario required to implement a network using high low mutation rate based routing protocol over different traffic density of nodes in network.

- Implementation of enhanced genetic algorithm and evaluation of their performance: in this simulation scenario the modified network protocol genetic algorithm is implemented and the performance of both the techniques are evaluated and compared.

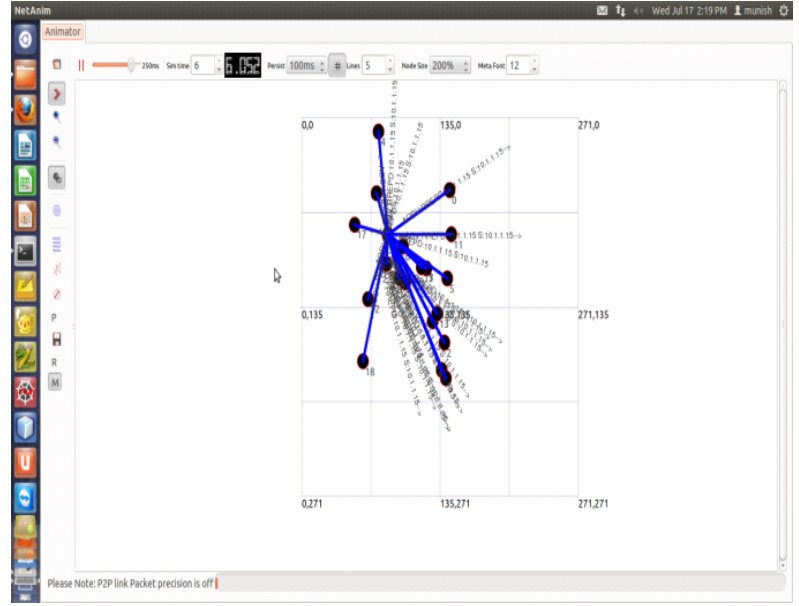

Figure 5: communications between nodes

\section{RESULTS AND COMPARATIVE ANALYSIS \\ 5.1Throughput}

Network throughput is sum of successful delivered data packets.The overall performance of the proposed hybrid genetic routing algorithm is much efficient than the traditional routing (high low hyper mutation genetic algorithm). The throughput is basically calculated in terms of bits per second and occasionally in terms of data packets per time slot or data packets per second.

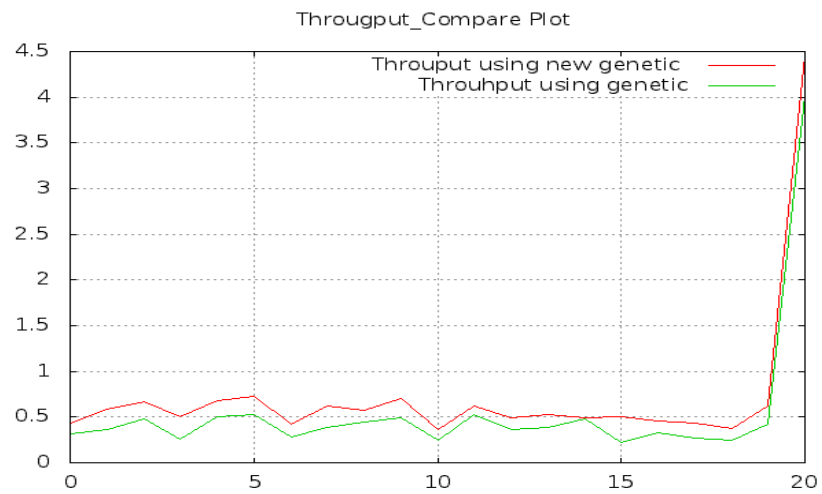

Figure 6: comparative throughput

In the above given figure 5.1 the performance comparison of enhanced hybrid routing and traditional high low mutation rate based method. Where the $\mathrm{X}$ axis indicates the number of nodes and $\mathrm{Y}$ axis provides the throughput. Graph shows the Network throughput in megabytes/sec with respect to number of nodes for both high low and hybrid genetic algorithm in the network.

In the figures red line indicates hybrid and green line indicate high low hyper mutation algorithm. It has seen that the throughput of genetic algorithm for 30 nodes is greater than high low hyper mutation genetic algorithm.

\subsection{Packet Delivery Ratio}

It is the ratio of the number of packets received at the destination with respect to the packet sent from the sources. PDR is estimated using the formula given: 


\section{Packet delivery ratio $=\frac{\text { Total deliwerd packets }}{\text { Total sent packets }}$}

Graph shows the Packet delivery ratio (PDR )in \% with respect to number of node. In the figure red line indicates hybrid approach and green line indicates high low mutation process. It has seen that the PDR of hybrid approach for 30 nodes is greater than high low mutation process.

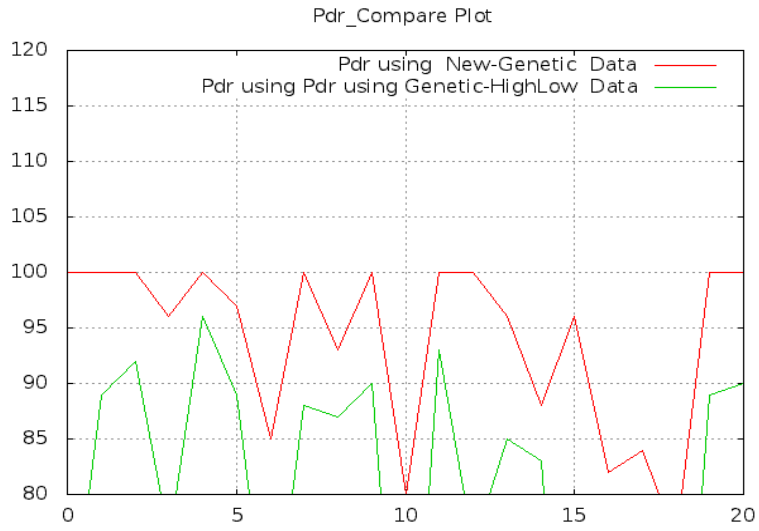

Figure 7: packet delivery ratios

\subsection{End to End Delay}

The total amount of time required to deliver a packet from source to destination is known as the end to end delay. Where $\mathrm{X}$ axis provides the number of nodes and $\mathrm{Y}$ axis provide the end to end delay in terms of milliseconds. The obtained comparative results for both routing algorithm is given using figure 5.3, where the proposed routing algorithm provides the less end to end delay as compare to traditional routing.

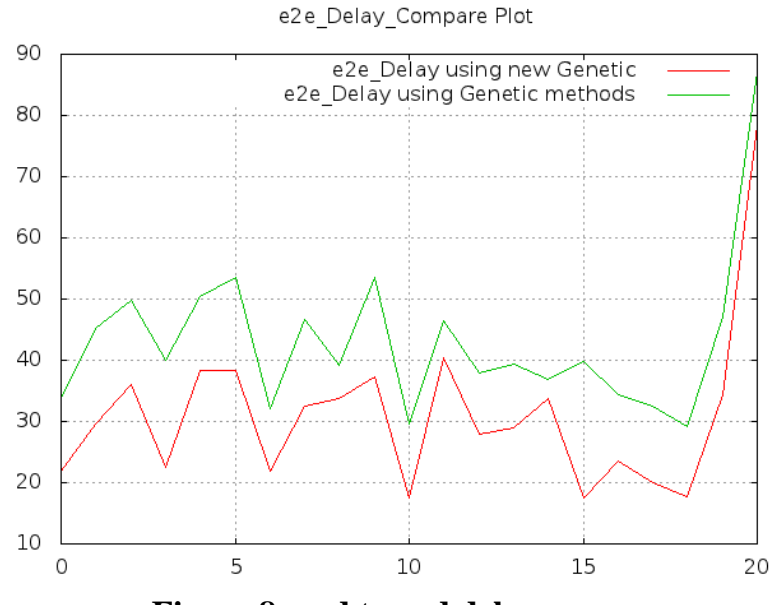

Figure 8: end to end delay

\subsection{Packet Drop Ratio}

Packet drop ratio demonstrates the amount of packet drop on number of nodes during communication. The comparison of packet drop ratio is established using the figure 5.4 where the $\mathrm{Y}$ axis indicates the amount of packet drop in percentage and the $\mathrm{X}$ axis provides the simulation time. According to the obtained results the genetic high low (green line) drops packet more frequently as compared with the proposed routing (hybrid routing) indicates red line technique.

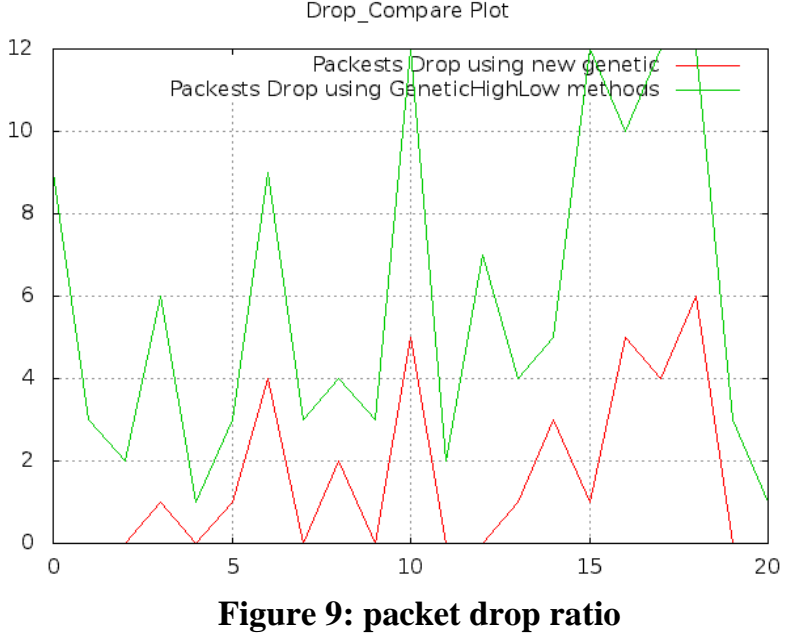

\section{CONCLUSION AND FUTURE WORK}

Routing is a backbone of the mobile ad hoc network. Routing algorithms are responsible for managing the topology of network. And also the shortest path discovery between source and destination is an essential task of routing protocol. The presented work is an investigation of dynamic multicast routing problem in mobile ad hoc network. For that purpose a new routing technique is proposed and implemented with small modification over the traditional genetic algorithm, for finding the best solution in a given search space, during the route discovery process is initiated of the routing approach.

The proposed routing (hybrid routing) technique is implemented using the NS3 network simulator. And the performance of the hybrid genetic routing algorithm is estimated and compared with the given high low mutation technique given in [1], that is also a kind of genetic algorithm technique derived from the changing in the mutation operator. According to the obtained results the performance of the proposed routing technique provides better performance as compared with the traditional approach [1].

Throughput of hlhmga is 0.4 , hybrid algorithm is 1 and packet delivery Ratio of hlhmga 95\% , hybrid $98 \%$ and Packets drop of hlhmga $25 \%$, hybrid 5\%. The comparative results show the effectiveness of the proposed routing strategy.

\section{Future Work}

Current research work has focused on efficiency parameters, throughput, packet delivery ratio and packets drop. But there is also the other efficiency parameters like power consumption, life time of network and packet size which can be studied in future work.

In future the proposed method can enhanced through using the change in fitness function and the cross over operator.

\section{ACKNOWLEDGMENTS}

With due respect I would like to inform you that the article which I had proposed for academic scenario. It is not feasible for industrial sector. In this article the references which I had taken from the listed one are not only the references. There are many other references which I had taken from the real world and from daily life aspect. 


\section{REFERENCES}

[1]Hui Cheng,Shengxiang Yang, "Hyper-mutation based Genetic Algorithms forDynamic Multicast Routing Problem in Mobile AdHoc Networks", 2012 IEEE 11th International Conference on Trust, Security and Privacy in Computing and Communications, 978-0-7695-4745-9/12 \$26.00 (C) 2012 IEEE, DOI 10.1109/TrustCom.2012.179

[2] Scalability study of the ad hoc on-demand distance vector routing protocol, INTERNATIONAL JOURNAL OF NETWORK MANAGEMENT 2003; 13: 97-114 (DOI: 10.1002/nem.463)

[3]KAPANG LEGO,PRANAV KUMAR SINGH,DIPANKAR SUTRADHAR, "Comparative Study of Adhoc Routing Protocol AODV, DSR and DSDV in Mobile AdhocNETwork",Indian Journal of Computer Science and Engineering Vol. 1 No. 4 364-371,

[4]Shiva Prakash,J. P. Saini,S. C. Gupta, "Methodologies and Applications of Wireless Mobile Ad-hoc Networks Routing Protocols", International Journal of Applied Information Systems (IJAIS) - ISSN : 2249-0868Foundation of Computer Science FCS, New York, USA Volume 1- No.6, February 2012

[5]TanuPreet Singh, Neha,Vikrant Das, "MULTICAST ROUTING PROTOCOLS INMANETS", International Journal of Advanced Research in Computer Science and Software Engineering, Volume 2, Issue 1, January 2012, ISSN: 2277 128X

[6]E. Bommaiah, M. Liu, A. McAuley, and R. Talpade, "AMRoute: Ad hocMulticast Routing Protocol", InternetDraft, draft-talpade-manet-amroute00.txt,August1998.

[7]C. W. Wu, Y. C. Tay, and C. K. Toh, "Ad hoc Multicast Routing ProtocolUtilizing Increasing id-numbers (AMRIS) Functional Specification",Internet-Draft, draft-ietf-manetamris-spec-00.txt, November 1998.

[8] Saaidal R. Azzuhri, Marius Portmann, Wee Lum Tan, "Evaluation of Parameterised Route Repair in AODV", Signal Processing and Communication Systems (ICSPCS), 2010 4th International Conference on,13-15 Dec. 2010

[9]Jyoti Jain,Roopam Gupta, T. K. Bandhopadhyay,“On Demand Local Link Repair Algorithm for AODV Protocol", International Journal of Computer Applications (0975 - 8887) Volume 35- No.5, December 2011

[10] R. K. Chauhan, ArzooDahiya, "AODV Extension using Ant Colony Optimization for Scalable Routing in VANETs", Journal of Emerging Trends in Computing and Information Sciences @2009-2012 CIS Journal. All rights reserved.

[11]Hemant Kumar Garg, P. C. Gupta,"Minimization of Average Delay, Routing Load and Packet Loss Rate in AODV Routing Protocol", International Journal of Computer Applications (0975 - 8887) Volume 44- No15, April 2012

[12] Kilhung Lee,"A backup path routing for guaranteeing bandwidth in mobile ad hoc networks for multimedia applications", Springer Science + Business Media, LLC 2011

[13] Dr.AdityaGoel,Ajaii Sharma,"Performance Analysis of Mobile Ad-hoc Network Using AODV Protocol", International Journal of Computer Science and Security (IJCSS), Volume (3): Issue (5) 2012
[14]Mrs.Smita A. Mungare, Dr.Rekha S. Patil,"REVIEW OF ENHANCED AODV ROUTING PROTOCOL FOR MOBILE AD HOC WIRELESS NETWORKS", ISSN: 2278 - 909X, International Journal of Advanced Research in Electronics and Communication Engineering (IJARECE), Volume 1, Issue 5, November 2012

[15] GENETIC ALGORITHMS FOR OPTIMIZATION, Programs for MATLAB ® Version 1.0 User Manual

[16]JifengXuan, He Jiang, ZhileiRen, "Pseudo Code of Genetic Algorithm and Multi-Start Strategy Based Simulated Annealing Algorithm for Large Scale Next Release Problem", Dalian University of Technology.

[17] Author: Shengxiang Yang ,Hui Cheng and Fang Wang, "Genetic Algorithms With Immigrants and Memory Schemes for Dynamic Shortest Path Routing Problems in Mobile Ad Hoc Networks" This Paper appears in: ieee transactions on systems, VOL. 40, NO. 1, Year of publication : JANUARY 2010.

[18] Author: G. Hossein EkbataniFard1*, Reza Monsefi2, Mohammad-R. Akbarzadeh-T.3, Mohammad H Yaghmaee4 "A Multi-objective Genetic Algorithm based Approach for Energy Efficient QoS-Routing in Two-tiered Wireless Sensor Networks" This Paper appears in: 5th International Symposium on Wireless Pervasive Computing (ISWPC), Year of publication : 2010.

[19] Author: G Felix Hoffmann, Daniel Medina , Adam Wolisz" Optimization of Routing and Gateway Allocation in Aeronautical Ad Hoc Networks Using Genetic Algorithms" This Paper appears in: In Proc. of the 7th International Wireless Communications and Mobile Computing Conference (IWCMC) 2011, Year of publication : 2011, Volume 0, PP. 1391-1396.

[20] Author: Glenn Leary, Krishnan Srinivasan, Krishna Mehta, and Karam S. Chatha, "Design of Network-on-Chip Architectures With a Genetic Algorithm-Based Technique" This Paper appears in: IEEE TRANSACTIONS ON VERY LARGE SCALE INTEGRATION (VLSI) SYSTEMS, Year of publication : 2009 VOL. 17, NO. 5.

[6] Author: Yi Mei, Student Member, IEEE, Ke Tang, Member IEEE, and Xin Yao, Fellow, IEEE " Decomposition-Based Memetic Algorithm for Multiobjective Capacitated Arc Routing Problem" This Paper appears in: IEEE IEEE TRANSACTIONS ON EVOLUTIONARY COMPUTATION, Year of publication : 2010.

[21] Author: Chrisy Samara, EiriniKarapistoli, and Anastasios A. Economides "Performance Comparison of MANET Routing Protocols based on real-life scenarios" This Paper appears in: Dept. of Information Systems University of Macedonia Thessaloniki, 54006 Greece, Year of publication : 2012.

[22] Author: Ashish Kumar, M. Q. Rafiq, and Kamal Bansal" Energy Efficient Routing Protocol Avoiding Route Breaks based on DSR" This Paper appears in:International Journal of Computer Applications (0975 - 8887), Volume 44-No.4, Year of publication : April 2012.

[23] Author: Tang, Maolin and Ai, Lifeng, A hybrid genetic algorithm for the optimal constrained web service selection problem in web service composition. This Paper appears in:Proceeding of the 2010 World Congress on Computational Intelligence,), Year of publication : 18-23 July 2010 . 